\title{
System for Evaluating Elements of VR Content that Cause Motion Sickness
}

\author{
Su-min Jung ${ }^{1}$, Dong-Yue Wang and Taeg-keun Whangbo* \\ ${ }^{1}$ Department of Computer Science Graduate School of IT, Gachon University, \\ 1342 Seongnamdaero, Sujeong-gu, Seongnam-si, Gyeonggi-do, Korea \\ *Gachon University, 1342 Seongnamdaero, Sujeong-gu, Seongnam-si, \\ Gyeonggi-do, Korea \\ ${ }^{1}$ sumini1019@nate.com, ${ }^{*}$ tkwhangbo@gachon.ac.kr
}

\begin{abstract}
With the rapid growth of the virtual reality (VR) market, global corporations have shown great interest in $V R$, and VR content has now become familiar to general users. However, most users suffer from difficulties in using VR content due to motion sickness. In this regard, this paper examines the definition, causes, and solutions of VR motion sickness and proposes a test system to prevent motion sickness in HMD-based VR content production. The VR Motion Sickness Inspection System uses the VR 360-degree image as an input to calculate VR motion sickness-inducing factors that determine the VR sickness risk of the content.
\end{abstract}

Keywords: VR, Human Factor, HMD, Motion Sickness

\section{Introduction}

Virtual reality (VR) content using head-mounted displays (HMDs) is being actively produced globally. VR is expanding its market not only in games and video contents but also in communications, electronics, and automobile industries. In addition, the formation of a market for the general public to produce films and games has led to the dissemination of massive amounts of contents. However, VR contents using HMD cause the users to go through various problems. Among them, motion sickness is considered to be the most serious problem. More than $60 \%$ of users who experience HMD-based VR for the first time feel motion sickness and perceive it as a very unpleasant experience [1].

For the HMD-based VR content market to continue to be successful, it is essential to prevent and solve motion sickness problems.

Human factor studies have been conducted to deal with motion sickness even in conventional three-dimensional (3D) research. As both 3D and VR images are based on the stereo image, elements of HMD-based VR content that cause motion sickness can be summarized based on the 3D human factor.

In this paper, elements that cause HMD-based VR motion sickness are specified, and VR content is basically implemented with stereo 360-degree image as a source. Image processing technologies are used to examine the contents of various VR devices, and a system that can check and prevent the risks of motion sickness caused by the stereo 360degree image-based content is proposed.

Received (February 15, 2018), Review Result (May 14, 2018), Accepted (May 28, 2018)

* Corresponding Author 


\section{Methods and Results}

\subsection{Definition and Causes of VR Motion Sickness}

In general, a human perceives gravity, horizontal-vertical rotation, etc., through the vestibular system in the inner ear that consists of otolith organs and semicircular canals. It is a general theory that motion sickness is caused by a discrepancy between gravitational and rotational information coming from the vestibular organs and visual information received from the eyes, and there is also an opinion that tactile information felt in the soles of the feet affects the occurrence of motion sickness [2].

The typical symptoms of motion sickness include vomiting and nausea due to the discrepancy between the direction of motion and that of gaze that people perceive when they see the interior of the automobile or aircraft while the actual body is moving [3].

There are two types of situations in which motion sickness occurs. First, visual information received through the eyes is insufficient while the sensory information of the vestibular organs changes. A case of car sickness is an example [4]. Second, there is a case where the change in sensory impulses that the vestibular organs convey is insufficient while the visual information changes. For example, a player is moving quickly in the VR content with HMD worn [5]. In this case, the visual information continues to change, but the player is sitting or standing still, which causes a discrepancy in information.

Likewise, the motion sickness that occurs when the user experiences VR content while wearing HMD is referred to as VR motion sickness, and it has emerged as the most difficult problem in VR market activation and content production.

\subsection{General Solutions for VR Motion Sickness}

As described above, the cause of VR motion sickness is the discrepancy that occurs when the visual information changes, but the sensory information of the vestibular organs does not change. There are two possible approaches to solve this problem. The first is to slow down the sense that enters the vestibular organs. The second is to match the visual information with the sensory information about equilibrium (the sense of balance).

The first approach is widely used in dealing with car sickness, and it is a method to reduce the sensation by paralyzing the vestibular system through the use of ear patches or medications. When used in the form of a drug for slowing down the senses of the vestibular system before the use of an HMD device, it can lower the incidence of motion sickness. However, this method is considered to be only a temporary solution because of a sense of rejection and the fear of side effects.

The second approach can be largely divided into three methods. They are the methods that forcibly change or alleviate the senses so that visual sensation can match sensory sensation as closely as possible.

- The first method is to inject information into a visual organ at the right time. It is the most widely used method in the current VR market. When the user changes the field of vision through head rotation while wearing the HMD, the matching screen is displayed without a delay. However, owing to hardware limitations, a latency of approximately $20 \mathrm{~ms}$ occurs between the user's head rotation and the change in the field of vision, based on a mid-range or high-end device, which serves the cause of motion sickness. Ultimately, a latency of $0 \mathrm{~ms}$ is required to prevent the occurrence of motion sickness. Graphic technology companies such as AMD and Nvidia are currently focusing on the development of a VR SDK, but the advancement of HMD device hardware seems to be the most plausible solution to the problem. 
- The second method is to inject information into a vestibular organ at the right time. Although there are few cases, the method has been continuously studied. Galvanic vestibular stimulation is a typical example of this method in which current flows through the vestibular organ to stimulate the sensation of the vestibular system and breaks the balance of the target in the desired direction. In the case of VR content, it is used in such a way as to stimulate the vestibular organs, forcibly break the physical balance of the player, and resolve the discrepancy. Samsung developed the Entrim 4D Project using this technology, but the project was abandoned for some reason.

- The third method is to change or minimize the width of visual information to enable a prediction and prevent a discrepancy between two sources of information. This method is about the matter of content planning and know-how, and thus it depends entirely on the ability of the planner. When unpredictable situations occur while the user is viewing VR content, the severity of motion sickness increases [6][7]. For example, when the player character moves excessively to the side or the back owing to a sudden shock, rapid changes in visual information can cause a discrepancy between the visual information and the sensory information of the vestibular organs, resulting in severe motion sickness.

\section{Proposed System}

\subsection{Necessity of System}

In the preceding sections, we examined the methods to prevent VR motion sickness. Ultimately, it is predicted that most VR motion sickness will disappear if hardware evolves to ensure that latency is lowered toward $0 \mathrm{~ms}$, resolution increases to $8 \mathrm{~K}$, and frame rates to 90-120 fps [8]. However, since expectations and demands for the VR market are spreading and increasing rapidly, much time is required to satisfy the ideal hardware.

Therefore, there is a need to devise a system that can minimize VR motion sickness in terms of planning and software. However, a failure to determine which elements of the content, other than the above hardware aspects, cause VR motion sickness forces a content producer to depend on the experience of the planner or the developer. In addition, the current VR motion sickness control program has a disadvantage in that it is not only costly and time-consuming to make an experiment but also has insufficient objectivity because it is only a measure to determine the risk of motion sickness by integrating the subjective dizziness of the contents through the subjects.

\subsection{System Overview}

The proposed system is designed to derive and warn of the risk of VR motion sickness by using VR stereo 360-degree image as a source, regardless of which HMD device the VR development environment is based on.
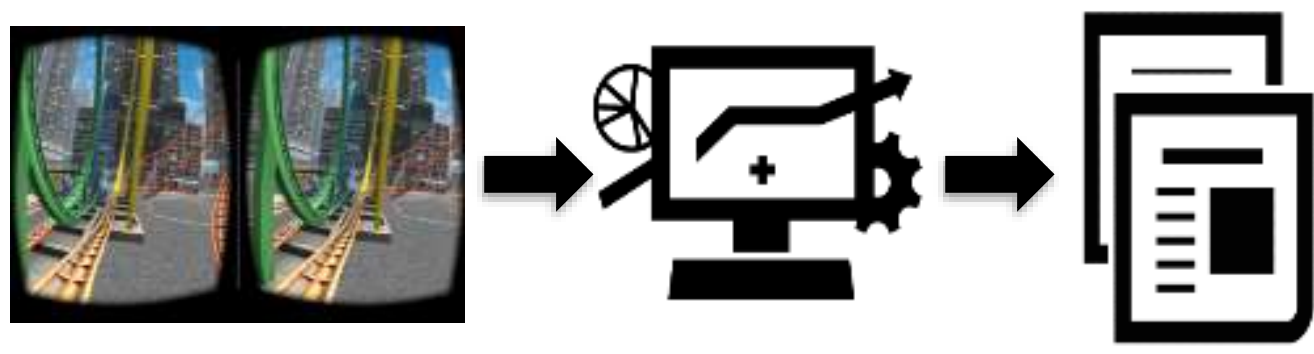

Figure 1. VR Motion Sickness Test System Overview 
The system calculates the risk of each element that causes VR motion sickness based on the image processing technology by inputting a VR stereo 360-degree image of content which is subject to testing the risk of VR motion sickness. If there is an element with a risk level above a certain value, the system reports the frame, the area where the problem occurred, and the elements that caused the motion sickness to a user. Through the process, the user can know whether there is a possibility of a specific time or area of the content causing VR motion sickness. Lastly, the system calculates the details of the problematic frame and the average risk of the entire image and then generates a report on the risk of VR motion sickness.

\subsection{Elements that Cause VR Motion Sickness}

To develop the proposed system, it is necessary to determine which phenomenon is responsible for causing motion sickness in VR content. The proposed system seeks to verify and calculate the risk by listing the elements that can be tested, based on the image processing technology, among factors inducing VR motion sickness that have not as yet been fully verified.

Table 1 lists the elements that cause VR motion sickness that are considered in the proposed system.

Table 1. Elements that cause VR Motion Sickness

\begin{tabular}{|c|l|}
\hline Causes of motion sickness & \multicolumn{1}{|c|}{ Detailed descriptions } \\
\hline $\begin{array}{c}\text { Excessive size of disparity } \\
\text { (absolute) }\end{array}$ & $\begin{array}{l}\text { The disparity of objects in the image is too large or } \\
\text { too small }\end{array}$ \\
\hline $\begin{array}{c}\text { Excessive size of disparity } \\
\text { (relative) }\end{array}$ & $\begin{array}{l}\text { The disparity of objects in the image becomes too } \\
\text { large or suddenly shrinks in a short period of time }\end{array}$ \\
\hline $\begin{array}{c}\text { Rapid movement of objects } \\
\text { Rapid movement of camera } \\
\text { viewpoint }\end{array}$ & Bulky main objects move quickly \\
\hline $\begin{array}{c}\text { Repeated frequent movements } \\
\text { of camera }\end{array}$ & $\begin{array}{l}\text { Camera shaking phenomenon } \\
\text { Camera shakes repeatedly and frequently when a } \\
\text { car runs on a road }\end{array}$ \\
\hline $\begin{array}{c}\text { Sudden movements of camera } \\
\text { to the side and rear }\end{array}$ & $\begin{array}{l}\text { Camera moves suddenly to the side-rear, not } \\
\text { forward }\end{array}$ \\
\hline
\end{tabular}

\subsection{Disparity-map Acquisition}

Among the elements that cause VR motion sickness, binocular disparity should be calculated from left eye-right eye images. In this case, a method for calculating a depth-map is very important in motion sickness testing since the reliability level of the disparity value is determined according to the accuracy of the depth-map [9].

In this paper, the depth-map was acquired using SGBM block matching algorithm, and the accuracy was improved by edge-based hole filling post-processing. The list of disparity-related elements that cause motion sickness in Table 1 is obtained from the acquired disparity values. 
$\mathbf{E}(\mathbf{D})=\sum_{p}\left(C(p, D p)+\sum_{q \in N p} P_{1} I\left[\left|D_{p}-D_{q}\right|=1\right]+\sum_{q \in N p} P_{2} I\left[\left|D_{p}-D_{q}\right|>1\right]\right)$

(1) is the SGBM algorithm. This algorithm aims to minimize the following global energy function $\mathrm{E}$ for disparity image $\mathrm{D}$.

With $P 2 \geq P 1$, where $E(D)$ is the energy for the disparity image, $D$ is $p$, and q represents indices for pixels in the image, $\mathrm{Np}$ is the neighborhood of the pixel $\mathrm{p}$. The function $\mathrm{C}(\mathrm{p}$, $\mathrm{Dp}$ ) is the cost of pixel matching with disparity in Dp. The parameter P1 is the penalty passed by the user for a change in disparity values of 1 between neighboring pixels. The parameter $\mathrm{P} 2$ is the penalty passed by the user for a change in disparity values greater than 1 between neighboring pixels. $\mathrm{I}[\mathrm{x}]$ is the function which returns 1 if the argument is true and 0 otherwise [10].

The minimized function produces a perfect disparity map with smoothing governed by parameters P1 and P2; however, minimizing the function for a two-dimensional (2D) image space is an NP-complete problem. The semi-global matching function approximates the 2D minimization by performing multiple one-dimensional (1D), or linear, minimizations [11]. The matching function aggregates costs on multiple paths which converge on the pixel under examination. Cost is computed for the disparity range specified by the minimum disparity and number of disparities parameters. By default, the matching algorithm aggregates costs for five directions [12]. You can set the full dynamic programming parameter to try to force the algorithm to aggregate costs for eight directions.

(a)
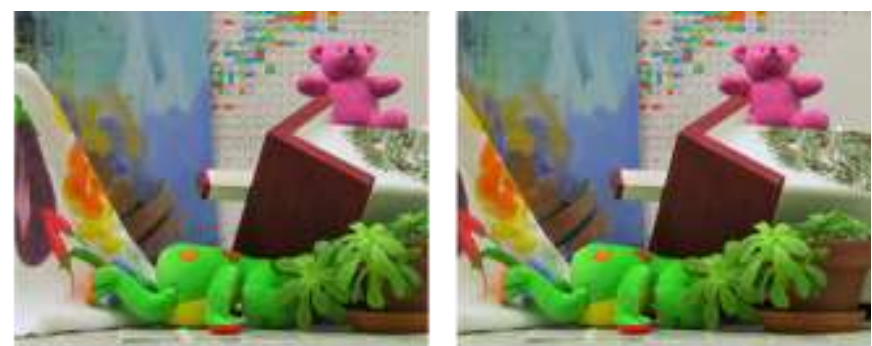

(b)
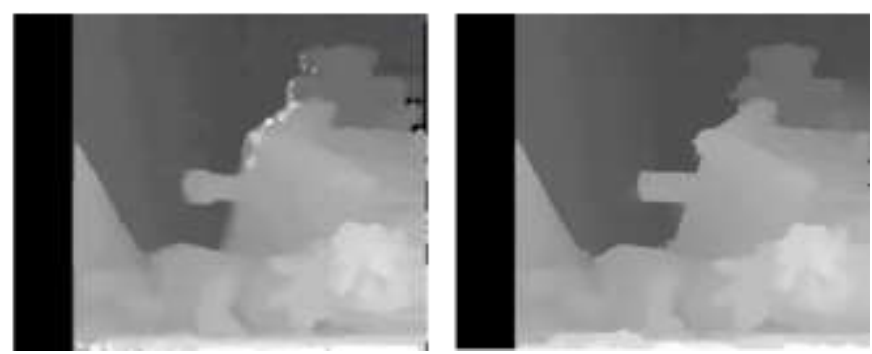

Figure 2. Disparity Map Acquisition Process

(a) : Left-Right Stereo Image

(b) : Before-After Hole Filling

\subsection{Image Correction According to 360-degree Image Features}

VR environments provide images with a 360-degree field of view for the user's immersion, and the images of sphere shape enhance the presence by changing the direction of the user's field of view with the camera viewpoint centered.

However, since the plane image is needed to acquire the aforementioned disparity map, a 360-degree image cannot be used in this case. To solve this problem, we use a 360-degree panoramic image with a plane structure, rather than a sphere-type 360-degree image. 


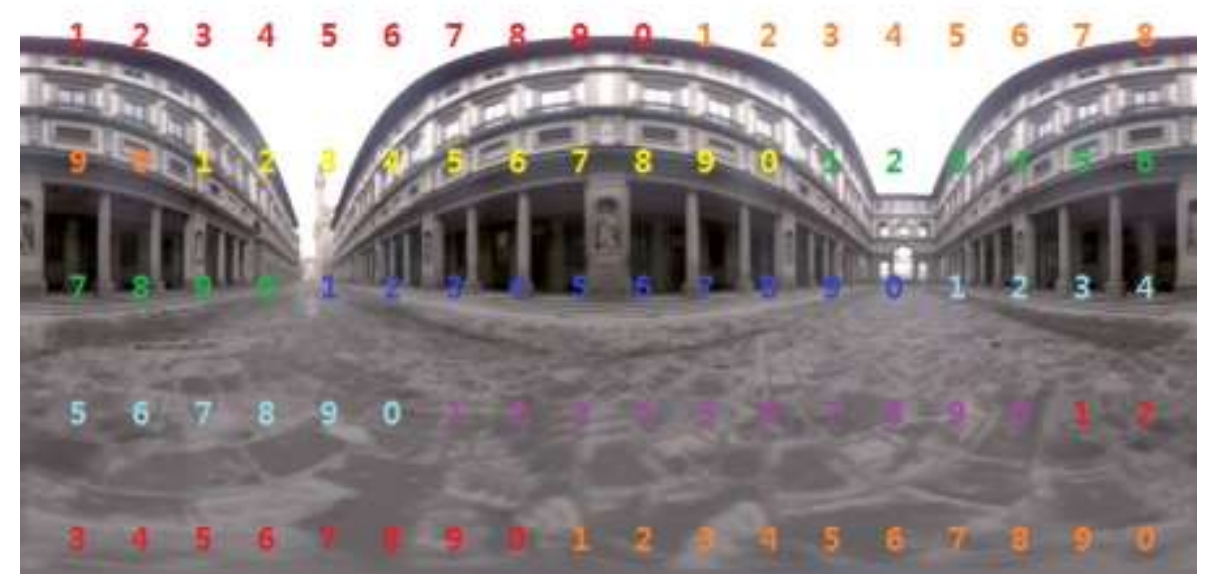

Figure 3. 360-degree Panorama Image

However, the 360-degree panoramic image has a noise problem that occurs in the disparity map acquisition step because there is a distortion in the image. To solve this problem, this study seeks to obtain a plane image without distortion by projecting the panoramic image onto a cube panorama with a distortion-free image in six directions. The obtained cubic image is inserted into the disparity map acquisition algorithm for each of the six directions, and each disparity map is acquired and merged to generate the final disparity map.

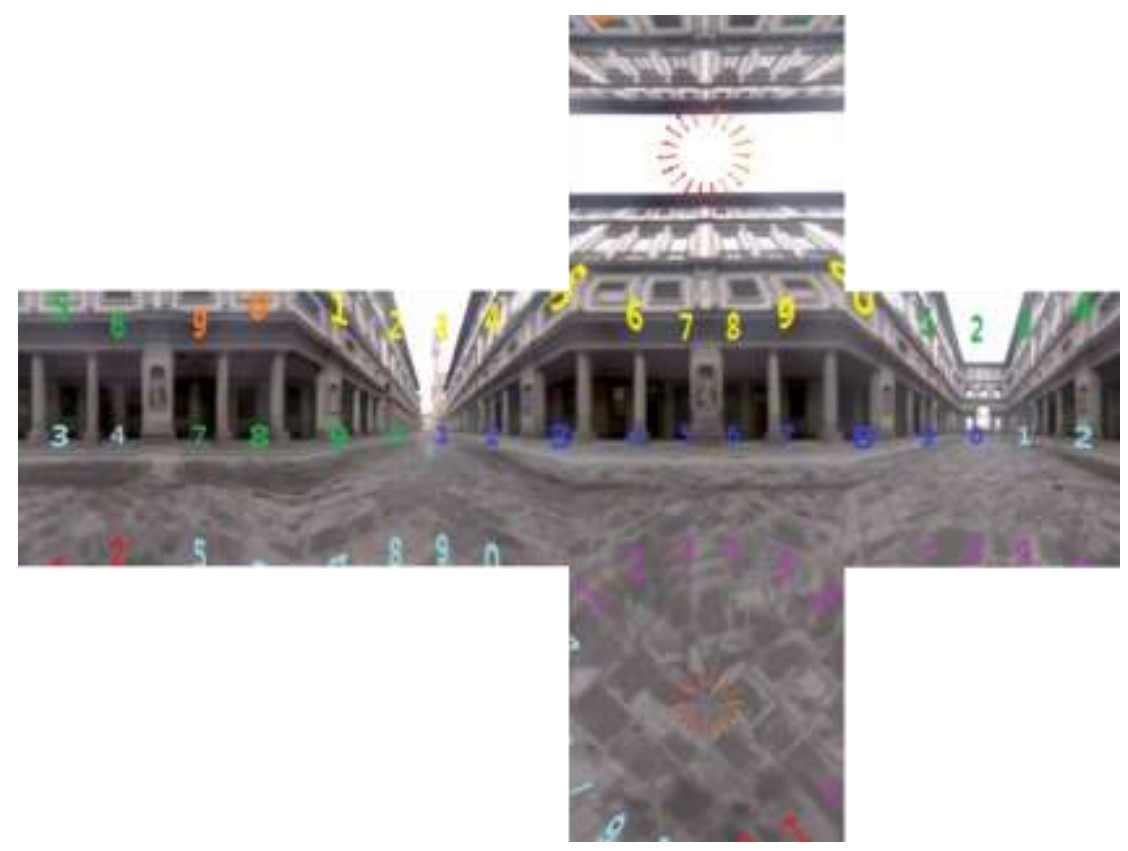

Figure 4. Converted Cubic Image

\subsection{System Configuration}

The proposed 360-degree image-based VR motion sickness test system measures the risk of VR motion sickness content in the following form.

The system receives the threshold of elements that cause VR motion sickness and a 360degree stereo image sequence as input from the user; it then calculates the motion sickness factor values for each frame from the start frame of the image sequence. If a motion sickness factor with a value higher than the threshold is found in the frame being examined, the system reports the information about the frame, goes to the next frame, and repeats the 
process. When checking the risk of motion sickness for each frame, the system examines the risk level of each factor in parallel based on the image processing. After all frames in the sequence are inspected, the final report is created, and the system is shut down.

The system user modifies the risk level of the motion sickness content by referring to the problematic frame-specific report and the final report.

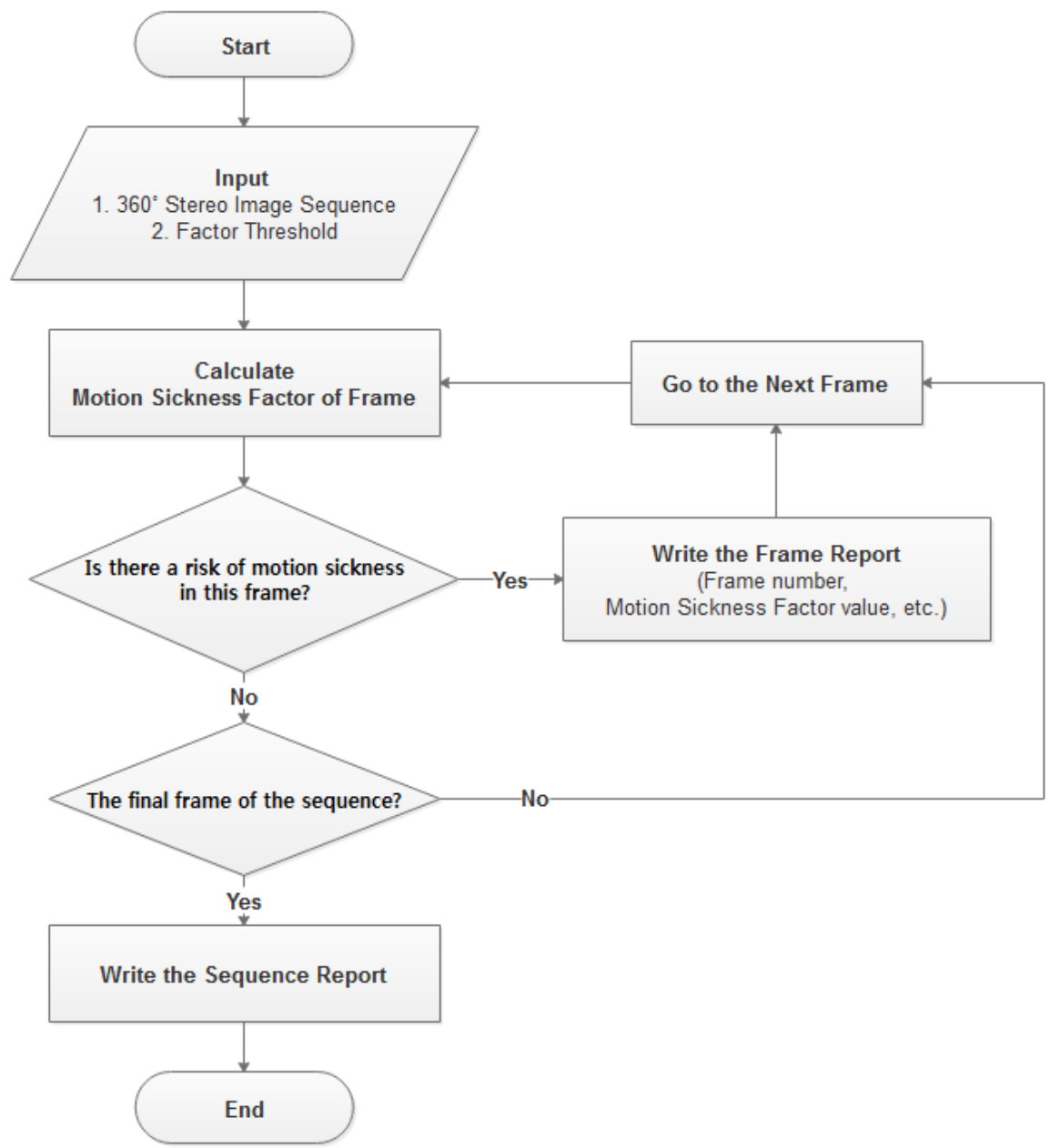

Figure 5. System Flowchart

\section{Conclusion}

Virtual reality is expanding its market day by day. HMD devices, starting with Oculus, are diversifying with the expansion of the mobile market, and various contents are being released accordingly. In this regard, this study sought to devise a system based on VR 360-degree stereo image to solve the big problem of motion sickness, regardless of various content environments. The use of the proposed system is expected to greatly improve the completeness of contents by preventing elements that can potentially cause motion sickness in VR content production.

Since few studies have been conducted regarding the field of VR motion sickness, content-related elements have not yet been fully verified except in regard to the hardware issue. For future research, there is a need for a clinical experiment to verify the elements that cause motion sickness considered in this paper and add other causes to improve the completeness of the research report. 


\section{Acknowledgments}

"This work was supported by the GRRC program of Gyeonggi province. [GRRCGachon2017(B03), Development of Personalized Digital Support Technology based on Artificial Intelligence]".

\section{References}

[1] SuperData, Report: Virtual Reality Consumers, (2017).

[2] L. J. Hettinger and G. E. Riccio, "Visually induced motion sickness in virtual environments", Presence: Teleoperators \& Virtual Environments, vol. 1, no. 3, (1992), pp. 306-310.

[3] J. Hakkinen, T. Vuori and M. Paakka, "Postural stability and sickness symptoms after HMD use", IEEE International Conference on Systems, Man and Cybernetics, vol. 1, (2002).

[4] S. M. Ebenholtz, "Motion sickness and oculomotor systems in virtual environments", Presence: Teleoperators \& Virtual Environments, vol. 1, no. 3, (1992), pp. 302-305.

[5] O. Merhi, "Motion sickness, console video games, and head-mounted displays", Human Factors: The Journal of the Human Factors and Ergonomics Society, vol. 49, no. 5, (2007), pp. 920-934.

[6] D. L. Harm, L. C. Taylor and J. J. Bloomberg, "Adaptive changes in sensorimotor coordination and motion sickness following repeated exposures to virtual environments", (2007).

[7] L. Rebenitsch and C. Owen, "Individual variation in susceptibility to cybersickness", Proceedings of the 27th annual ACM symposium on User interface software and technology, ACM, (2014).

[8] M. J. Potel, "Motion Sick in Cyberspace", IEEE Computer Graphics and Applications, vol. 18, no. 1, (1998).

[9] J. Cox, S. L. Hingorani, S. B. Rao and B. M. Maggs, “A maximum likelihood stereo algorithm”, Computer Vision and Image Understanding, vol. 63, (1996) May, pp. 542-567.

[10] L. Alvarez, "Dense disparity map estimation respecting image discontinuities: A PDE and scale-space based approach", Journal of Visual Communication and Image Representation, vol. 13, no. 1-2, (2002), pp. 3-21.

[11] S. Bing Kang, R. Szeliski and J. Chai, "Handling occlusions in dense multi-view stereo", Computer Vision and Pattern Recognition, 2001. CVPR 2001. Proceedings of the 2001 IEEE Computer Society Conference on IEEE, (2001), p. I-I.

[12] D. Scharstein and R. Szeliski, "A taxonomy and evaluation of dense two-frame stereo correspondence algorithms", International journal of computer vision, vol. 47, no. 1-3, (2002), pp. 7-42.

\section{Authors}

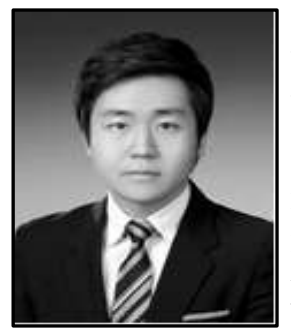

Su-min Jung received a bachelor's degree in 2013 from Gachon University, Korea, and a master's degree in 2015 from Gachon University, Korea. Mr. Jung has been working as an algorithm developer for one year in a vision and image processing company in Korea. He is currently pursuing a Ph.D. in computer engineering from Gachon University, Korea. His research areas include image processing, deep learning, healthcare service, and AR/VR/MR.

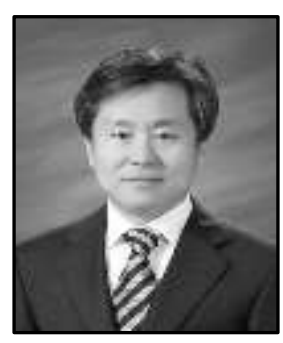

Taeg Keun Whangbo received a M.S. degree in computer science from City University of New York, USA in 1988 and a Ph.D. in computer science from Stevens Institute of Technology, USA in 1995. Currently, Mr. Whangbo is a professor in the Department of Interactive Media, Gachon University, Korea. He is also the Dean of Research Affairs at Gachon University. Before he joined Gachon University, Mr. Whangbo was a software developer at Q-Systems, Inc., New Jersey, USA from 1988 to 1993. He was also a researcher at Samsung Electronics, Korea from 2005 to 2007. From 2006 to 2008, Mr. Whangbo was the president of the Association of Korea Cultural Technology. His research areas include computer graphics, HCI, and AR/VR/MR. 